\title{
QCD Effective action at high temperature and small chemical potential
}

\author{
C. Villavicencio and E. S. Fraga
}

Instituto de Física, Universidade Federal do Rio de Janeiro, C.P. 68528, Rio de Janeiro, RJ 21941-972, Brazil.

We present a construction of an effective Yang-Mills action for QCD, from the expansion of the fermionic determinant in terms of powers of the chemical potential at high temperature for the case of massless quarks. We analyze this expansion in the perturbative region and find that it gives extra spurious information. We propose for the non-perturbative sector a simplified effective action which, in principle, contains only the relevant information.

\section{Introduction}

There has been an increasing interest in the few last years in the sign problem or phase problem in QCD. For finite chemical potential, the fermion determinant matrix is nonpositive definite, so it is not possible to perform Monte-Carlo simulations [1, 2]. The Glasgow method [3] and rewheighting techniques [4] have made great advances in the description of phase transitions on the lattice, considering a set of parameters near the transition line. However, the problem is still far from being solved.

There is special interest in the region of high temperature and low chemical potential, since it corresponds to the high-energy heavy-ion collision scenario. In this regime it is possible to expand the fermion determinant in powers of $\mu / T[$ [5, 6, 7]. This kind of expansion is what we are interested in investigating. As one performs this expansion, some questions arrise: what order is enough to cut the expansion? which is its range of validity? how to distinguish between real and complex terms? Differently from what is done in the previously mentioned papers, we keep the expanded series as a functional of the gauge fields, giving rise to an effective non-local Yang-Mills action.

\section{Basic idea of the expansion}

For simplicity, consider one-flavor massless quarks. The QCD generating functional at finite chemical potential in euclidean space is defined as

$\mathcal{Z}=\int \mathcal{D} G \operatorname{det}\left(-i \not D+i \mu \gamma_{4}\right) e^{-S_{\mathrm{YM}}[G]}$,

where $G$ are the gluon fields, also present in the covariant derivative $D$, and $S_{\mathrm{YM}}$ is the Yang-Mills action. We can expand the fermion determinant in powers of the chemical potential assuming that $\mu<\Lambda \sim T$ : 


$$
\begin{aligned}
& \operatorname{det}\left(-i \not D+i \mu \gamma_{4}\right) \\
& \quad=\operatorname{det}(-i \not D) \exp \left\{-\sum_{s=1}^{\infty} \frac{(i \mu)^{s}}{s} \int_{Y_{1} \cdots Y_{s}} \operatorname{Tr} \gamma_{4} S\left(Y_{2}, Y_{1}\right) \gamma_{4} S\left(Y_{3}, Y_{2}\right) \ldots \gamma_{4} S\left(Y_{1}, Y_{n}\right)\right\}
\end{aligned}
$$

where $S\left(Y_{b}, Y_{a}\right)$ is the dressed fermion propagator, which can be expressed as a series in powers of the gauge field and the free fermion propagator. The expansion, then, will contribute to additional terms in the Yang-Mills effective action

$S_{\mathrm{YM}}^{\mathrm{eff}}=S_{\mathrm{YM}}+\sum_{n=0}^{\infty} \int_{X_{1} \ldots X_{n}} \Gamma_{{ }_{a_{1} \ldots a_{n}}^{(n)}{ }^{\mu_{1} \ldots \mu_{n}}}\left(X_{1}, \ldots, X_{n}\right) G_{\mu_{1}}^{a_{1}}\left(X_{1}\right) \ldots G_{\mu_{n}}^{a_{n}}\left(X_{n}\right)$,

where the vertices $\Gamma^{(n)}$ are series in powers of the chemical potential: $\Gamma^{(n)}=\sum_{s \geq 1} \Gamma^{(n, s)}$, with $\Gamma^{(n, s)} \sim \mu^{s}$. In this way we have a positive-definite fermion determinant and the contribution from the chemical potential will be part of an effective Yang-Mills action. The problem now is to find criteria to cut this infinite series in terms of the chemical potential and gauge fields.

\section{Perturbative QCD}

To test the expansion, we study it firstly in the perturbative sector. Setting $G \rightarrow g G$, we find naturally the way to cut the gauge field series, which is given by the order of perturbative corrections. For one-loop corrections, we just need the effective action up to order $g^{2}$, so

$S_{\mathrm{YM}}^{\mathrm{eff}}=S_{\mathrm{YM}}+\Gamma^{(0)}+g^{2} \int_{X Y} \Gamma_{\mu \nu}^{(2)^{a b}}(X, Y) G_{\mu}^{a}(X) G_{\nu}^{b}(Y)$.

We calculate, then, the pressure up to order $g^{2}$. Considering the expansion on chemical potential up to order $\mu^{4}$, we surprisingly obtain the same result as in the usual pertubative QCD calculation with the chemical potential included in the quark propagator [8].

However, the next terms in the chemical potential series are non-zero. In particular, the next vacuum contribution for the pressure is

$P_{0}^{(6)}=-\frac{\Gamma^{(0,6)}}{\beta V} \approx 0.9334 N_{c} \frac{\mu^{6}}{\pi^{2} T^{2}}$

which is negligible only for $\mu \ll \Lambda$. More than establishing the range of validity, this result shows that this kind of expansion contains some spurious information, at least for the massless case, and in principle the only indication on where to cut the series is the dimension of the desired observable.

\section{Non-perturbative QCD}

For the non-perturbative regime, we need another criterium to cut the series. The most appropriate is the Weinberg power counting [9], i.e. considering the external momentum as a small parameter, $p<\Lambda$, and also assuming that the chemical potential and the 
expectation value of the fields are of the same order, $\mu \sim G \sim p$. This gives a low-energy theory where the effective Lagrangian can be expanded in soft modes. In the case of Yang-Mills theories, this soft-mode expansions for high temperatures correspond to the Hard Thermal Loop (HTL) approximation [10, 11]. The minimal action must be of order $p^{4}$. So, applying the power counting, the minimal effective action is

$S_{\mathrm{YM}}^{\min }[G, \mu]=S_{\mathrm{YM}}[G]+S^{(0,2)}(\mu)+S^{(0,4)}(\mu)+S_{\mathrm{LO}}^{(2,2)}[G, \mu]+S_{\mathrm{LO}}^{(3,1)}[G, \mu]$,

where the indices $(n, s)$ denote powers of $n$ in the gauge field and $s$ in the chemical potential, and LO means leading order in the HTL approximation. In this case, the appearance of imaginary terms will happen for $S_{\mathrm{LO}}^{(3,1)}$.

The whole series of gauge fields is gauge invariant in every order in the $\mu$ expansion, i.e. $\sum_{n} S^{(n, s)}$ is gauge invariant for all $s$. In the case of the minimal effective action, the contributions $S^{(2,2)}$ and $S^{(3,1)}$ are independently gauge invariant, and HTL preserves this feature.

Of course this effective action must be tested, but in principle it could provide rich information on the confinement-deconfinement transition line.

\section{Acknowledgements}

We thanks Phillipe de Forcrand for comments and suggestions. We acknowledge financial support from CAPES, CLAF, CNPq, FAPERJ and FUJB-UFRJ.

\section{REFERENCES}

1. S. Hands, Nucl. Phys. Proc. Suppl. 106 (2002) 142.

2. E. Laermann and O. Philipsen, Ann. Rev. Nucl. Part. Sci. 53 (2003) 163.

3. I.M. Barbour and A.J. Bell, Nucl. Phys. B 372 (1992) 385.

4. Z. Fodor and S.D. Katz, Phys. Lett. B 534 (2002) 87.

5. S.A. Gottlieb, W. Liu, D. Toussaint and R.L. Renken, R.L. Sugar, Phys. Rev. Lett. 59 (1987) 2247.

6. C.R. Allton, S. Ejiri, S.J Hands, O. Kaczmarek, F. Karsch, E. Laermann and C. Schmidt, Phys. Rev. D 68 (2003) 014507.

7. C.R. Allton, M. Doring, S. Ejiri, S.J. Hands, O. Kaczmarek, F. Karsch, E. and K. Redlich, Phys. Rev. D 71 (2005) 054508.

8. J.I. Kapusta, Finite Temperature Field Theory, Cambridge University Press, 1989.

9. S. Weinberg, Physica A 96 (1979) 327.

10. E. Braaten and R.D. Pisarski, Phys. Rev. D 45 (1992) 1827.

11. J. Frenkel and J.C. Taylor, Nucl. Phys. B 374 (1992) 156. 